\title{
QUADRATIC PAIRS FOR ODD PRIMES
}

\author{
BY C. Y. HO
}

Communicated by R. M. Fossum, June 6, 1976

The purpose of this note is to announce a result in the classification of the quadratic pair for odd prime where the group is quasisimple. The complete proof can be found in [4] and [5]. For the convenience of the reader the terminology particular to this area is recollected here. A finite group, $X$, is quasisimple if $X$ is perfect and $X / Z(X)$ is simple, where $Z(X)$ is the center of $X$. Let $M$ be a vector space over the field $K$ and $G$ a subgroup of the general linear group of $M$. Let $Q=\left\{g \in G \backslash\{1\} \mid M(g-1)^{2}=0\right\}$. We say that $(G, M)$ is a quadratic pair if $M$ is an irreducible $K G$ module and $G$ is generated by $Q$. Let $p$ be an odd prime. We say that $(G, M)$ is a quadratic pair for $p$ if $K$ is the field of $p$ elements and the dimension of $M$ over $K$ is finite. Let $d=\min _{\sigma \in Q}\{\operatorname{dim} M(\sigma-1)\}$ and $Q_{d}=$ $\{\tau \in Q \mid \operatorname{dim} M(\tau-1)=d\}$. For each $\sigma \in Q_{d}$, let $E(\sigma)=\{\tau \in Q \mid M(\sigma-1)=$ $M(\tau-1)$ and $\left.C_{M}(\sigma)=C_{M}(\tau)\right\} \cup\{1\}$. Then $E(\sigma)$ is an elementary abelian $p$ subgroup of $G$. Let $\Sigma=\left\{E(\sigma) \mid \sigma \in Q_{d}\right\}$. We say that $(G, M)$ is a quadratic pair for 3 whose root group has order 3 if $|E|=3$ for any $E \in \Sigma$.

Lemma 4.1 of [3] gives the following result. Let $(G, M)$ be a quadratic pair for 3 whose root group has order 3. Let $\sigma, \tau \in Q_{d}$. Then $\langle\sigma, \tau\rangle$ is isomorphic to one of the following groups: (a) $S L(2,3)$, (b) $S L(2,5)$, (c) $S L(2,3) \times Z_{3}$, (d) $Z_{3}$ or $Z_{3} \times Z_{3}$, (e) the nonabelian 3-group of order 27, exponent 3 and nilpotent class 2 .

THEOREM. Let $(G, M)$ be a quadratic pair for $p, p$ odd, such that $G$ is quasisimple. If $(G, M)$ is a quadratic pair for 3 whose root group has order 3, then we also assume that for some $E \in \Sigma$, the set $\{F \mid F \in \Sigma$ and $\langle E, F\rangle \cong$ $\left.S L(2,3) \times Z_{3}\right\}$ is empty. Under these conditions $G / Z(G)$ is isomorphic to one of the following groups.

(1) Groups of Lie type of odd characteristic: $A_{n}(q)(n \geqslant 2$ except in the case $q=3$ where we have $n \geqslant 3),{ }^{2} A_{n}(q)(n \geqslant 2), B_{n}(q)(n \geqslant 3), C_{n}(q)(n \geqslant 2)$, $D_{n}(q)(n \geqslant 3),{ }^{2} D_{n}(q)(n \geqslant 3),{ }^{3} D_{4}(q), G_{2}(q), F_{4}(q), E_{6}(q),{ }^{2} E_{6}(q), E_{7}(q)$ where $q=p^{b}$ for some positive integer $b$.

(2) Alternating groups: $A_{n}, n \geqslant 5$.

(3) Groups of Lie type of even characteristic: $P G U_{n}(2), S p(6,2), D_{4}(2)$, $G_{2}(4)$.

(4) Sporadic groups: $\mathrm{HJ}, \mathrm{Suz}, \mathrm{Co}{ }_{1}$.

Furthermore we have $p=3$ whenever (2), (3) or (4) holds.

AMS (MOS) subject classifications (1970). Primary 20D05, 20G40; Secondary $20 \mathrm{H} 30$. 
Some more technical definitions are needed for the sketch of the proof of the theorem. Let $(G, M)$ be a quadratic pair for an odd prime $p$ such that $G$ is quasisimple. For $X \in \Sigma$, let $I(X)=\{j \mid$ there is $Y$ in $\Sigma$ such that $j$ is an involution of $\langle X, Y\rangle$. An involution $i$ of $I(X)$ is long if $\operatorname{dim} M(i-1)=2 d$, otherwise $i$ is short. It can be shown easily that if there exists a short involution, then $p=$ 3 and $(G, M)$ is a quadratic pair for 3 whose root group has order 3 .

We now sketch the proof of the theorem. First we treat the case where $I(E)$ consists of long involutions only. Let $i \in I(E)$. A subnormal subgroup $H$ of the centralizer of $i$ is constructed such that $i$ belongs to $H$ and $H$ is isomorphic to one of the following groups: (a) $S L(2, q)$, where $q=p^{b}$ with $b \geqslant 1$; (b) $\hat{A}_{n}$, the perfect 2-fold covering of the alternating group $A_{n}$ with $n \geqslant 5$. If $i$ belongs to the center of $G$, then the proof is trivial. If $i$ does not belong to the center of $G$, then we apply results in [1], [2] and [6] to obtain the possibilities for $G / Z(G)$ and argue that some possibilities cannot happen. It is shown that $G / Z(G)$ is isomorphic to one of the groups listed in (1) or (2) of the theorem. Thus we may assume that $(G, M)$ is a quadratic pair for 3 whose root group has order 3 in the rest of the argument. If $\{F \mid F \in \Sigma$ and $\langle E, F\rangle \cong S L(2,5)\}$ is empty, then [1] completes the proof. Suppose there exists $F \in \Sigma$ such that $\langle E, F\rangle \cong S L(2,5)$. Let $i$ be the involution of $\langle E, F\rangle$. Then one of the following holds.

(1) The centralizer of $i$ in $G$ contains a subnormal subgroup $H$ such that $i \in H$ and $H \cong \hat{A}_{n}$ for some $n \geqslant 4\left(\hat{A}_{4} \cong S L(2,3)\right)$.

(2) $G / Z(G) \cong P G U_{n}(2)$.

(3) $i$ is the unique long involution of $G$.

If (1) holds, then similar argument as before shows the theorem holds in this case. If (2) holds, then the theorem holds. Suppose finally (3) holds. For any subset $S$ of $G$ let $\bar{S}=S Z(G) / Z(G)$. Let $E=\langle e\rangle$ and let $\bar{e}=b$. In $\bar{G}$ let $D$ be the conjugacy class of elements of order 3 containing $b$. Then the subgroup of $\bar{G}$ generated by two noncommutating elements in $D$ is isomorphic to one of the following groups: $S L(2,3), A_{4}, A_{5}$. We can now apply results in [7] to get the possibilities for $G / Z(G)$. After showing that some of the possibilities cannot happen the proof of the theorem is then complete. We remark that the $\Delta$ operator introduced in [8] is very useful in manipulations. As pointed out in [5] the basic idea of this theorem comes from [8].

The author would like to take this opportunity to thank the International Bureau of GMD for their support during the author's stay in Tübingen. He also thanks Professor Hering and the Department of Mathematics in Tübingen.

\section{REFERENCES}

1. M. Aschbacher and M. Hall, Jr., Groups generated by a class of elements of order 3, J. Algebra 24 (1973), 591-612. MR 47 \#327.

2. M. Aschbacher, A characterization of certain Chevalley groups and its application to component type groups (unpublished). 
3. C. Y. Ho, On the quadratic pair whose root group has order 3, Bull. Inst. Math. Acad. Sinica 1 (1973), no. 2, 155-180. MR 49 \#9080. appear).

4. Chevally groups of odd characteristic as quadratic pairs, J. Algebra (to

5. - On the quadratic pairs, J. Algebra (to appear).

6. R. Solomon, Finite groups with intrinsic 2-component of type $\hat{A}_{n}$, J. Algebra 33 (1975), 498-522. MR 51 \#8251.

7. B. Stellmacher, Einfache Gruppen die von einer Konjugiertenklasse von Elementen der Ordnung drei erzeugt werden, J. Algebra 30 (1974), 320-354. MR 50 \#10054.

8. J. G. Thompson, Quadratic pairs (unpublished). BRASÍLIA, BRASIL

DEPARTMENTO DE MATEMÁTICA, UNIVERSIDADE DE BRASÍLIA, 70,000, 